\title{
Scrofula as a presentation of tuberculosis and HIV
}

\author{
Katrina Barnett, MD;* Ron Medzon, MD†
}

\begin{abstract}
Scrofula, or tuberculous cervical lymphadenitis, though now rare, is more commonly seen in minorities, women and immunosuppressed patients, especially those with HIV. We discuss a patient who presented to the emergency department with an anterior neck abscess and was diagnosed with both advanced HIV and disseminated tuberculosis. A high level of suspicion is necessary to make this diagnosis, but given an increasing degree of global mobility, such patients may present anywhere. Medical management is effective, though difficult. Early diagnosis improves the patient's individual prognosis and may prevent further exposure and transmission to the population.

\section{RÉSUMÉ}

Même si elle est rare de nos jours, la scrofule ou lymphadénite cervicale tuberculeuse se manifeste plus souvent chez les minorités, chez les femmes et chez les patients immunodéprimés, et en particulier ceux qui sont infectés par le VIH. Nous décrivons le cas d'un patient qui s'est présenté à l'urgence avec un abcès à la partie antérieure du cou et chez lequel on a diagnostiqué à la fois une infection avancée au VIH et une tuberculose miliaire. Il faut avoir de solides raisons de soupçonner cette maladie pour poser un tel diagnostic, mais compte tenu de la mobilité mondiale croissante, ces patients peuvent se présenter n'importe où. La prise en charge médicale est efficace, mais difficile. Le diagnostic hâtif améliore le pronostic individuel du patient et peut prévenir une exposition plus grave et la transmission à la population.
\end{abstract}

\section{Introduction}

About $15 \%$ of cases of tuberculosis (TB) present with extrapulmonary disease, and of those roughly $50 \%$ are centred in the lymph nodes. Scrofula, or tuberculous cervical lymphadenitis, makes up about $60 \%$ of these cases of TB. Although rare, such presentations are more common in women, minorities and immunocompromised patients, especially those with HIV. ${ }^{1,2}$ HIV and TB are the most prevalent infectious global killers, and their presence in the same individual is even more deadly., Since TB can spread rapidly within an immunocompetent population, suspecting its presence is imperative to protecting hospital staff and the population at large. We present a case of a neck abscess that was the initial presentation of both advanced HIV and disseminated TB.

\section{Case presentation}

A 28-year-old woman presented to the emergency department (ED) with the chief complaint of a neck abscess. She arrived in the United States from Cape Verde (off the coast of west Africa) 2 days before this presentation. She stated that the abscess had started 3 weeks earlier as a small pimple, but that it gradually worsened. Since then, she had experienced fevers as high as $102^{\circ} \mathrm{F}\left(38.9^{\circ} \mathrm{C}\right)$, and had developed a productive cough over the previous 2 weeks. She reported that she had been treated within the last month with a week-long course of amoxicillin and an incision and drainage. Upon further questioning, she stated that her husband had HIV but that she did not, and that she had recently had a negative Purified Protein Derivative test (PPD) before coming to the United States. The initial

\footnotetext{
*Resident Physician of Emergency Medicine and †Assistant Professor of Emergency Medicine, Boston Medical Center, Boston University School of Medicine, Boston, Mass.
}

Received: June 19, 2006; revisions received: Dec. 4, 2006; accepted: Dec. 11, 2006

This article has been peer reviewed.

Can J Emerg Med 2007;9(3):176-9 
history was obtained with the translation assistance of a family member.

Upon physical examination, the patient was welldeveloped, ill-appearing and in mild distress. Her vital signs were: blood pressure $100 / 59 \mathrm{~mm} \mathrm{Hg}$, heart rate 114 beats/min, temperature $99.1^{\circ} \mathrm{F}\left(37.3^{\circ} \mathrm{C}\right)$, respiratory rate 18 breaths/min, and oxygen saturation of $100 \%$ on room air. The patient also had oral thrush. Her neck exam revealed a $14 \mathrm{~cm} \times 12 \mathrm{~cm}$ warm, erythematous, raised, fluctuant mass at the junction of the right anterior neck and the clavicle with evidence of purulent tracking superiorly. She had an active wet cough, but clear breath sounds. She was tachycardic and had a grade III/IV systolic murmur at the left sternal border radiating to the axilla. The remainder of the physical exam was unremarkable.

A chest $\mathrm{x}$-ray showed a left upper lobe infiltrate. A CT scan of the chest revealed a focal consolidation with cavitation in the left upper lobe as well as multiple miliary pulmonary nodules and mediastinal abscesses. A CT scan of the neck revealed a $7.5 \mathrm{~cm} \times 6.8 \mathrm{~cm}$ multiloculated right neck abscess extending from the sixth cervical vertebrae to the clavicle, and complete thrombosis and occlusion of the right internal jugular vein (Fig. 1). There were multiple enlarged lymph nodes in the right cervical chain, measuring up to $2.6 \mathrm{~cm}$. Laboratory tests revealed a white blood cell count of 9800 with $88 \%$ neutrophils, $10 \%$ bands and a hematocrit of $20 \%$.

After viewing the chest $\mathrm{x}$-ray, the patient was moved to a negative pressure room and TB precautions were taken (i.e., the patient wore a surgical mask, and the staff wore 1860S, N95 particulate respirator masks made by $3 \mathrm{M})$. During her ED stay, the patient spiked a fever of $105^{\circ} \mathrm{F}\left(40.5^{\circ} \mathrm{C}\right)$, and her systolic blood pressure dropped to the mid-80s $(\mathrm{mm} \mathrm{Hg})$. She remained conscious, alert and asymptomatic, but her blood pressure remained persistently low despite

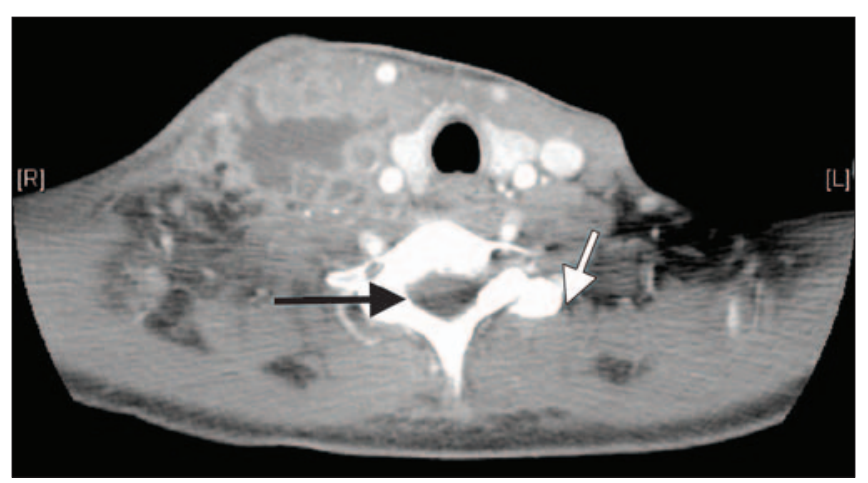

Fig. 1. CT scan of the neck with contrast. The black arrow indicates the multiloculated abscess. The white arrow indicates the right internal jugular vein with a tiny ring of contrast around the occluding thrombus.
$4 \mathrm{~L}$ of intravenous saline and she was admitted to the medical intensive care unit for management of possible septic shock. Pressors were not started in the ED as the patient remained asymptomatic despite consistent blood pressure readings below $100 \mathrm{~mm}$ systolic. Three sets of blood cultures were drawn and sent from the ED. Given the systolic murmur and potential for endocarditis, the patient was started on nafcillin and gentamycin.

The patient spent 16 days in the hospital. The otolaryngology and cardiothoracic surgery services were consulted initially about her neck abscess, but given its size, location and the likelihood of a chronic fistulous tract, they decided to simply perform a needle aspiration and treat the patient medically. That aspirate grew out $4+$ acid fast bacilli, as did her sputum. Her $C D 4$ count was $2 / \mathrm{mL}$, and her HIV viral load was 500000 copies $/ \mathrm{mL}$. Her antibiotic coverage was broadened to include vancomycin and cefepime as she continued to spike fevers through the initial antibiotics. The patient was also started on 4-drug therapy for tuberculosis (rifampin, isoniazid, pyrazinamide and ethambutol). The patient responded well, and by the time of discharge the abscess was no longer raised, erythematous or fluctuant. Despite the worry of endocarditis raised by her heart murmur, her transthoracic echocardiogram showed no vegetations and only mild mitral and tricuspid regurgitation. The cultures eventually grew Mycobacterium tuberculosis complex. The patient's blood pressure continued to be intermittently low throughout her stay, despite copious hydration. The etiology was unclear, but ultimately it was felt to be more likely to be due either to HIV nephropathy or neuropathy, as opposed to sepsis. Upon further questioning using a hospital translator, the patient admitted to previous knowledge of her HIV diagnosis through testing in Cape Verde, though she was not aware that she had TB. Her case was reported to the local infection control officer, the local Center of Disease Control, and the head of the Communicable Disease Department for the city of Boston. Medical staff had a repeat of their PPD at 3 months postexposure, and the information was also forwarded to the Federal Aviation Administration for further follow-up of the patient's contacts.

\section{Discussion}

Scrofula, or tuberculous cervical lymphadenitis, is an old diagnosis. While the primary site of infection in TB is the lungs, in up to $15 \%$ of cases an extrapulmonary site may produce the first presenting symptoms. ${ }^{1}$ Lymphadenitis is the most common extrapulmonary presentation of $\mathrm{TB}$, and the cervical region is the most common site $(63 \%$ of all 
tuberculous lymphadenitis in one study). ${ }^{1}$ Lymph node involvement arises from either hematogenous or lymphatic spread from the lungs. ${ }^{1,5}$ Extrapulmonary TB is more common in children, women and minorities (for unclear reasons) as well as in patients who are immunosuppressed, especially those with HIV. ${ }^{2,3}$ In patients with HIV, this form of TB often accompanies disseminated $\mathrm{TB}^{2}$ and correlates with declining $C D 4$ counts, presumably because the impaired immune system cannot contain the mycobacteria in a pulmonary Ghon complex. ${ }^{6,7}$ As the $C D 4$ count drops below $100 / \mathrm{mL}$, up to $70 \%$ of patients with HIV and TB may manifest extrapulmonary disease. ${ }^{8}$ As seen in our case, cervical lymphadenitis was the patient's first presentation of both her TB and her HIV, despite the fact that she already had occult disseminated disease. Any bulky disease process can affect the surrounding structures by its mass alone. In this case, there was thrombosis, though not collapse, of the internal jugular vein in the area of the tuberculoma. The vessel recannulated when the disease retreated under therapy. In the literature, there are several case reports and descriptions of vascular thrombosis and collapse related to TB, both in the cervical region and in the pulmonary vessels where it has even mimicked pulmonary embolism. ${ }^{9,10}$

Tuberculosis and HIV are the 2 most common infectious killers in the world. There were an estimated 8.3 million new cases of TB in 2000, with $9 \%$ attributable to HIV. However, in certain parts of the world (Africa as well as the United States), co-infection rates can reach 30\%. ${ }^{1}$ Patients with HIV are more susceptible to TB, and TB accelerates patients' development of HIV. ${ }^{3}$ However, unlike many of the diseases that affect patients with HIV-compromised immune systems, TB can also spread rapidly among an immunocompetent population. ${ }^{4}$ Barnes's work on the fingerprinting of specific TB strains in Los Angeles located the source patient, who was not HIV positive but who lived in close quarters with many others in homeless shelters. ${ }^{11}$

Another complicating factor in the convergence of HIV and TB is that the standard screening method for TB, skin testing with a PPD, may fail in a patient with HIV who is anergic. Anergy, or immune unresponsiveness, is correlated with decreasing $C D 4$ count, especially when the $C D 4$ count is below $200 / \mathrm{mL}^{12}$ Our patient's recent negative PPD was likely a false negative due to her advanced HIV status. If TB is highly suspected in an HIV patient, repeat testing may help augment the response, but even this is not always reliable. ${ }^{12}$ At that point, culture and acid fast staining of sputum or needle aspirations (in the case of extrapulmonary disease) should be used to help make the diagnosis.

Treatment of HIV and TB should focus first on the TB, especially when both are new diagnoses. Both diseases require a rigorous multidrug treatment regimen, which may produce multiple interactions, especially in the first few months of TB treatment. Current recommendations are to first control the acute TB, and once compliance has been established, start HIV therapy. ${ }^{3}{ }^{13}$ Occasionally, a patient started on antiretroviral therapy while being treated for TB will experience a worsening of their TB symptoms, or even develop new symptoms. This is owing to immune reconstitution syndrome in which the regenerating immune system produces a stronger inflammatory response at the sites of infection, paradoxically making the patient more symptomatic. ${ }^{13}$

TB rarely requires surgical intervention, even in bulky extrapulmonary disease. As seen in our case, medical therapy was rapidly effective in reducing the size of the abscess, as well as the internal jugular thrombosis. Extrapulmonary TB is treated with $6-12$ months of medical therapy, depending on the site. Cervical lymphadenitis is adequately treated with a 6-month course, but CNS, bone and joint disease require a longer regimen., ${ }^{2,13,14}$ The World Health Organization recommends directly observed therapy for TB as the most effective treatment given the complexity and prolonged nature of the course.

Finally, it is important to remember that global migration is now routine. New immigrants may require a higher vigilance for disease presentations that may be uncommon in local populations. ${ }^{15}$ While the United States does not require a PPD in visitors, immigrants are required to undergo medical screening, including a PPD. This case once again highlights how the complete medical history may not be revealed by a non-English speaking patient who must rely on a family member for translation, especially when discussing a diagnosis of HIV, which has historically been stigmatized. If at all possible, professional translation should be used to obtain the most accurate history, not only for that patient's benefit, but also for the benefit of the staff and those who have come in contact with the patient.

\section{Conclusion}

Scrofula is no longer a common syndrome, but with a growing number of immunocompromised patients, particularly those with HIV, it is an important diagnosis to consider. As in our case, tuberculous lymphadenitis may be the presenting symptom for both HIV and TB. The overlap and interaction of TB and HIV has proven to be a deadly combination and though treatment can be effective, it is also complex and demanding. Increased global migration means patients with these diagnoses may appear in any $\mathrm{ED}$, and a high index of suspicion is crucial for early 
diagnosis. This affects both the patient's prognosis and the risk of exposure posed to health care personnel and the population at large.

Competing interests: None declared.

\section{References}

1. Geldmacher H, Taube C, Kroeger C, et al. Assessment of lymph node tuberculosis in northern Germany: a clinical review. Chest 2002;121:1177-82.

2. Mandell GL, Dolin R, eds. Douglas, and Bennett's principles and practice of infectious diseases. 6th ed. Philadelphia (PA): Elsevier; 2005.

3. Barnes PF, Lakey DL, Burman WJ. Tuberculosis in patients with HIV infection. Infect Dis Clin North Am 2002;16:107-26.

4. Corbett EL, Watt CJ, Walker N, et al. The growing burden of tuberculosis: global trends and interactions with the HIV epidemic. Arch Intern Med 2003;163:1009-21.

5. Frieden TR, Sterling TR, Munsiff SS, et al. Tuberculosis. Lancet 2003;362:887-99.

6. Shriner KA, Mathisen GE, Goetz MB. Comparison of mycobacterial lymphadenitis among persons infected with human immunodeficiency virus and seronegative controls. Clin Infect Dis 1992;15:601-5.

7. Lee MP, Chan JW, Ng KK, et al. Clinical manifestations of tuberculosis in HIV-infected patients. Respirology 2000;5:423-6.
8. Jones BE Young SM, Antoniskis D, et al. Relationship of the manifestations of tuberculosis to CD4 cell counts in patients with human immunodeficiency virus infection. Am Rev Respir Dis 1993;148:1292-7.

9. King AD, Ahuja AT, Metreweli C. MRI of tuberculous cervical lymphadenopathy. J Comput Assist Tomogr 1999;23:244-7.

10. Drake WM, Elkin SL, al-Kutoubi A, et al. Pulmonary artery occlusion by tuberculous mediastinal lymphadenopathy. Thorax 1997;52:301-2.

11. Barnes PF, Yang Z, Pogoda JM, et al. Foci of tuberculosis transmission in central Los Angeles. Am J Respir Crit Care Med 1999;159:1081-6.

12. Huebner RE, Schein MF, Hall CA, et al. Delayed-type hypersensitivity anergy in human immunodeficiency virus-infected persons screened for infection with mycobacterium tuberculosis. Clin Infect Dis 1994;19:26-32.

13. de Jong BC, Israelski DM, Corbett EL, et al. Clinical management of tuberculosis in the context of HIV infection. Annu Rev Med 2004;55:283-301.

14. van Loenhout-Rooyackers JH, Laherj RJ, Richter C, et al. Shortening the duration of treatment for cervical tuberculous lymphadenitis. Eur Respir J 2000;15:192-5.

15. Weis SE, Burgess G. Tuberculosis control in a border state. Treatment of the foreign-born. Infect Dis Clin North Am 2002;16:59-71.

Correspondence to: Dr. Katrina Barnett, Dowling 1 South, 1 Boston Medical Center Place, Boston MA 02118; katrina.barnett@gmail.com 